\title{
Note on the Lower Bound of Least Common Multiple
}

\author{
Shea-Ming Oon \\ Institute of Mathematical Sciences, University of Malaya, 50603 Kuala Lumpur, Malaysia \\ Correspondence should be addressed to Shea-Ming Oon; oonsm@um.edu.my \\ Received 1 July 2012; Revised 24 December 2012; Accepted 10 January 2013 \\ Academic Editor: Pekka Koskela \\ Copyright (C) 2013 Shea-Ming Oon. This is an open access article distributed under the Creative Commons Attribution License, \\ which permits unrestricted use, distribution, and reproduction in any medium, provided the original work is properly cited. \\ Consider a sequence of positive integers in arithmetic progression $u_{k}=u_{0}+k r$ with $\left(u_{0}, r\right)=1$. Denote the least common multiple \\ of $u_{0}, \ldots, u_{n}$ by $L_{n}$. We show that if $n \geqslant r^{2}+r$, then $L_{n} \geqslant u_{0} r^{r+1}(r+1)$, and we obtain optimum result on $n$ in some cases for such \\ estimate. Besides, for quadratic sequences $m^{2}+c,(m+1)^{2}+c, \ldots, n^{2}+c$, we also show that the least common multiple is at least \\ $2^{n}$ when $m \leqslant\lceil n / 2\rceil$, which sharpens a recent result of Farhi.
}

\section{Introduction}

Integer sequences in arithmetic progressions constitute a recurrent theme in number theory. The most notable result in this new century is perhaps the existence of arbitrary long sequences of primes in arithmetic progressions due to Green and Tao [1].

The bounds of the least common multiple for the finite sequences in arithmetic progressions also attract some attention. The prime number theorem assures that the least common multiple of the first $n$ positive integers is asymptotically upper bounded by $(e+\varepsilon)^{n}$ and lower bounded by $(e-\varepsilon)^{n}$ for any prefixed $\varepsilon$. As for effective uniform estimate, Hanson [2] obtained the upper bound $3^{n}$ about forty years ago by considering Sylvester series of one. Nair [3] gave $2^{n}$ as alower bound in a simple proof ten years later in view of obtaining a Chebyshev-type estimate on the number of prime numbers as in a tauberian theorem due to Shapiro [4].

Recently, some results concerning the lower bound of the least common multiple of positive integers in finite arithmetic progressions were obtained by Farhi [5]. Some other results about the least common multiple of consecutive integers and consecutive arithmetic progression terms are given by Farhi and Kane [6] and by Hong and Qian [7], respectively. If $a_{0}, \ldots, a_{n}$ are integers, we denote their least common multiple by $\left[a_{0}, \ldots, a_{n}\right]$. Consider two coprime positive integers $u_{0}$ and $r$, and put $u_{k}=u_{0}+k r, L_{k}=\left[u_{0}, \ldots, u_{k}\right]$. A recent result of Hong et al. (cf. $[8,9]$ ) shows that for any positive integers $a, \alpha, r$, and $n$ such that $a \geqslant 2, \alpha, r \geqslant a, n \geqslant 2 \alpha r$, we have $L_{n} \geqslant u_{0} r^{\alpha+a-2}(r+1)^{n}$.
Recently, Wu et al. [10] improved the Hong-Kominers lower bound. A special case of Hong-Kominers result tells us that if $n \geqslant r(r+3)$ (or $n \geqslant r(r+4))$ if $r$ is odd (or even), then $L_{n} \geqslant u_{0} r^{r+1}(r+1)^{n}$. In this note, we find that the HongKominers lower bound is still valid if $n \in\left[r(r+1), r\left(r+r_{0}\right)\right)$ with $r_{0}=3$ or 4 if $r$ is odd or even. That is, we have the following result.

Theorem 1. Let $n, u_{0}, r \in \mathbb{N}$ with $\left(u_{0}, r\right)=1$. One puts for any $k \in \llbracket 0, n \rrbracket, u_{k}=u_{0}+k r$ and $L_{n}=\left[u_{0}, \ldots, u_{n}\right]$. Then, for any $n \geqslant r(r+1)$,

$$
L_{n} \geqslant u_{0} r^{r+1}(r+1)^{n}
$$

Furthermore, if $u_{0}>r$ or $u_{0}<\min \{3, r\}$, the same estimate holds when $n=r^{2}+r-1$.

In 2007, Farhi [11] showed that $\left[1^{2}+1,2^{2}+1, \ldots, n^{2}+\right.$ $1] \geqslant 0.32(1.442)^{n}$. Note that Qian et al. [12] obtained some results on the least common multiple of consecutive terms in a quadratic progression. We can now state the second result of this paper.

Theorem 2. Let $c, m, n \in \mathbb{N}$ be such that $0<m<n$. Suppose that $m \leqslant\lceil n / 2\rceil$. Then, one has

$$
\left[m^{2}+c,(m+1)^{2}+c, \ldots, n^{2}+c\right] \geqslant 2^{n} \text {. }
$$

This theorem improves the result in [11]. 


\section{Proof of the First Theorem}

Let $x, y \in \mathbb{R}$ with $y \neq 0$. We say that $x$ is a multiple of $y$ if there is an integer $z$ such that $x=y z$. As usual, $\lfloor x\rfloor$ denotes the largest integer not larger than $x$, and $\lceil x\rceil$, denotes the smallest integer not smaller than $x$.

We will introduce the two following results. The first is a known result which tells us that $L_{n}$ is a multiple of $\left(u_{0} \cdots u_{n} / n !\right)$. This is can be proved by considering a suitable partial fraction expansion (cf. [11]) or by considering the integral $\int_{0}^{1} x^{u_{0} / r-1}(1-x)^{n} d x$ (cf. [13]).

For $\ell \in \llbracket 0, n \rrbracket$, with a slight modification of notation as in [11], we put $L_{n, \ell}=\left[u_{n-\ell}, \ldots, u_{n}\right]$ and

$$
B_{n, \ell}:=\frac{u_{n-\ell} \cdots u_{n}}{\ell !} .
$$

Clearly, we have $L_{n}=L_{n, n}$ and for any $\ell \in \llbracket 0, n \rrbracket, L_{n} \geqslant L_{n, \ell}$. This result can be restated as

Lemma 3. For any $\ell \in[0, n]$, one can find a positive integer $A_{n, \ell}$ such that $L_{n, \ell}=A_{n, \ell} B_{n, \ell}$.

Our modification aims to emphasize the estimate of the terms $A_{n, \ell}$ and $B_{n, \ell}$ that will give us some improvement. If $n \geqslant u_{0}$, then we see that the first term $u_{0}$ does not play an important role as it will be a factor of the term $u_{0}+u_{0} r=$ $u_{0}(1+r)$. Hence, the behaviour should be different when $n$ is large. It will be more interesting to give a control over the last $\ell$ terms.

Note that $B_{n, \ell+1}=\left(u_{n-\ell-1} /(\ell+1)\right) B_{n, \ell}$, thus

$$
\begin{aligned}
B_{n, \ell+1} & \leqslant B_{n, \ell} \Longleftrightarrow u_{0}+(n-(\ell+1)) r \leqslant \ell+1 \Longleftrightarrow \ell+1 \\
& \geqslant \frac{n r+u_{0}}{r+1} .
\end{aligned}
$$

We will put $\ell_{n}=\min \left\{\left\lfloor u_{n} /(r+1)\right\rfloor, n\right\}$. The following lemma tells us that keeping the first smaller terms can increase at least the power $n$ in the estimate of lower bound in such a way (cf. also [13]).

Lemma 4. One has $B_{n, \ell_{n}} \geqslant u_{0}(r+1)^{n}$.

Proof. We can just proceed by mathematical induction.

If $n=0$, then $\ell_{n}=0$ and $B_{0,0}=u_{0}$, and it holds. In fact, if $n \leqslant u_{0}$, then we have $\ell_{n}=n$ and

$$
B_{n, n}=u_{0}\left(\frac{u_{0}}{1}+r\right) \cdots\left(\frac{u_{0}}{n}+r\right) \geqslant u_{0}(1+r)^{n} .
$$

When $n \geqslant u_{0}$, we have $\left(n r+u_{0}\right) /(r+1) \leqslant(n r+n) /(r+1) \leqslant$ $n$ and $\ell_{n}=\left\lfloor\left(n r+u_{0}\right) /(r+1)\right\rfloor$. It remains to derive the result for the case $n+1$ from that of $n \geqslant u_{0}$.

It is obvious that $\ell_{n} \leqslant \ell_{n+1} \leqslant \ell_{n}+1$ and $u_{n} /(r+1)-1 \leqslant$ $\ell_{n} \leqslant u_{n} /(r+1)$.

If $\ell_{n}=\ell_{n+1}$, then $(n+1) r+u_{0} \leqslant\left(\ell_{n}+1\right)(r+1)$ and $u_{n-\ell_{n}}=u_{0}+\left(n-\ell_{n}\right) r \leqslant \ell_{n}+1$.

Thus,

$$
\frac{u_{n+1}}{u_{n-\ell_{n}}}=\frac{u_{n-\ell_{n}}+\left(\ell_{n}+1\right) r}{u_{n-\ell_{n}}}=1+\frac{\ell_{n}+1}{u_{n-\ell_{n}}} r \geqslant 1+r .
$$

Hence,

$$
B_{n+1, \ell_{n+1}}=\frac{u_{n+1}}{u_{n-\ell_{n}}} \cdot B_{n, \ell_{n}} \geqslant u_{0}(r+1)^{n+1} .
$$

If $\ell_{n+1}=\ell_{n}+1$, then

$$
B_{n+1, \ell_{n+1}}=\frac{u_{n+1}}{\ell_{n}+1} \cdot B_{n, \ell_{n}} \geqslant u_{0}(r+1)^{n+1} .
$$

In either case, the principle of mathematical induction assures the result. Then,

We can complete our proof now. Suppose that $n \geqslant r(r+1)$.

$$
\ell_{n}=\left\lfloor\frac{u_{n}}{r+1}\right\rfloor \geqslant\left\lfloor r^{2}+\frac{u_{0}}{r+1}\right\rfloor \geqslant r^{2}
$$

By considering the first $r$ multiples of $r$, we have $r^{r+1} \mid \ell_{n}$ !. Since $\left(r, u_{0}\right)=1$, we deduce that for all $k \in \llbracket 0, n \rrbracket,\left(r, u_{k}\right)=1$. Writing the result of Lemma 3 for $\ell=\ell_{n}$ as

$$
\ell_{n} ! \cdot L_{n, \ell_{n}}=A_{n, \ell_{n}} \cdot u_{n-\ell_{n}} \cdots u_{n},
$$

we conclude that $r^{r+1} \mid A_{n, \ell_{n}}$.

Using the Lemma 4, we obtain

$$
L_{n} \geqslant L_{n, \ell_{n}}=A_{n, \ell_{n}} B_{n, \ell_{n}} \geqslant u_{0} r^{r+1}(r+1)^{n},
$$

which is our conclusion.

Consider the case $n=r^{2}+r-1$. If $u_{0}>r$, then we still have $\ell_{n} \geqslant r^{2}$.

Supposing now that $r \geqslant 2$ and $u_{0} \leqslant \min \{2, r-1\}$, we shall prove that, for $n_{0}=r^{2}+r-1$, it is still possible to choose $\ell_{n_{0}}^{\prime}=r^{2}$ and $n_{0}-\ell_{n_{0}}^{\prime}=r-1$ so that $B_{n_{0}, \ell_{n_{0}}^{\prime}} \geqslant u_{0}(r+1)^{n_{0}}$.

On the one hand, when $n=r^{2}-r-1$, we have

$$
\begin{aligned}
\ell_{n} & =\left\lfloor\frac{u_{0}+r\left(r^{2}-r-1\right)}{r+1}\right\rfloor \\
& =\left\lfloor\frac{r^{2}(r+1)-2 r(r+1)+r+u_{0}}{r+1}\right\rfloor \\
& =r^{2}-2 r+\left\lfloor\frac{r+u_{0}}{r+1}\right\rfloor \\
& =r^{2}-2 r+1 .
\end{aligned}
$$

Thus, $n-\ell_{n}=r-2$ and

$$
\frac{u_{r-2} \cdots u_{r^{2}-r-1}}{\left(r^{2}-2 r+1\right) !} \geqslant u_{0}(r+1)^{r^{2}-r-1} \text {. }
$$

On the other hand, we write

$$
\begin{aligned}
B_{n_{0}, \ell_{n_{0}}^{\prime}}= & \frac{u_{r-1} \cdots u_{r^{2}+r-1}}{r^{2} !} \\
= & \frac{u_{r-2} \cdots u_{r^{2}-r-1}}{\left(r^{2}-2 r+1\right) !} \cdot \frac{u_{r^{2}-r} u_{r^{2}}}{u_{r-2}\left(r^{2}-r+1\right)} \\
& \cdot \prod_{s=1}^{r-1} \frac{u_{r^{2}-r+s} u_{r^{2}+r-s}}{\left(r^{2}-2 r+s+1\right)\left(r^{2}-(s-1)\right)} .
\end{aligned}
$$


It suffices to show that for any $s \in \llbracket 1, r-1 \rrbracket$,

$$
\begin{gathered}
\frac{u_{r^{2}-r+s} u_{r^{2}+r-s}}{\left(r^{2}-2 r+s+1\right)\left(r^{2}-(s-1)\right)} \geqslant(r+1)^{2}, \\
\frac{u_{r^{2}-r} u_{r^{2}}}{u_{r-2}\left(r^{2}-r+1\right)} \geqslant(r+1)^{2} .
\end{gathered}
$$

Equation (15) is equivalent to

$$
u_{r^{2}-r+s} u_{r^{2}+r-s} \geqslant\left(r^{2}-2 r+s+1\right)\left(r^{2}-(s-1)\right)(r+1)^{2} .
$$

By expanding, it remains to verify for any integer $r \geqslant s+$ $1 \geqslant 2$ that

$$
f_{s}(r):=2 u_{0} r^{3}-(4 s-1) r^{2}+2 s(s-1) r+s^{2}+u_{0}^{2}-1 \geqslant 0 .
$$

With simple computation, we obtain for any real $r, s>0$ with $r \geqslant s+1$,

$$
\begin{aligned}
f_{s}(s+1)= & 2\left(u_{0}-1\right) s^{3}+6\left(u_{0}-1\right) s^{2} \\
& +2\left(3 u_{0}-2\right) s+u_{0}^{2}+2 u_{0} \geqslant 0, \\
f_{s}^{\prime}(r) \geqslant & 6 r^{2}-2(4 s-1) r+2 s(s-1) \\
\geqslant & 2(r-s)(3 r-s+1) \geqslant 0,
\end{aligned}
$$

which allow to establish (15).

Equation (16) is equivalent to

$$
\begin{aligned}
& \left(u_{0}+\left(r^{2}-r\right) r\right)\left(u_{0}+r^{3}\right) \\
& \geqslant\left(u_{0}+(r-2) r\right)\left(r^{2}-r+1\right)(r+1)^{2}
\end{aligned}
$$

or

$$
\begin{gathered}
\left(2-u_{0}\right) r^{4}+\left(u_{0}-1\right) r^{3}+\left(1-u_{0}\right) r^{2} \\
+\left(2-u_{0}\right) r+u_{0}^{2}-u_{0} \geqslant 0
\end{gathered}
$$

Such inequality holds for any positive integer $r$ when $u_{0}=$ 1 or 2.

Finally, we still have

$$
B_{n_{0}, \ell_{n_{0}}^{\prime}} \geqslant u_{0}(r+1)^{n_{0}} .
$$

and the condition $\ell_{n_{0}}^{\prime} \geqslant r^{2}$ allows us to conclude.

\section{Proof of the Second Theorem}

We shall start by proving the following lemma.

Lemma 5. Let $c, m, n \in \mathbb{N}$ be such that $0<m<n$. Put

$$
L_{m, n}^{\prime}:=\left[m^{2}+c,(m+1)^{2}+c, \ldots, n^{2}+c\right] .
$$

Then,

$$
L_{m, n}^{\prime} \geqslant \frac{\prod_{k=m}^{n} \sqrt{k^{2}+c}}{(n-m) !} .
$$

Proof. We shall denote $x^{\imath}=\cos (\log x)+\imath \sin (\log x)$.

Consider the integral of complex-valued function of a real variable

$$
\int_{0}^{1} x^{m-1+\sqrt{c} l}(1-x)^{n-m} d x
$$

Firstly, by integrating by parts $(n-m)$ times,

$$
\begin{aligned}
\int_{0}^{1} x^{m-1+\sqrt{c} l}(1-x)^{n-m} d x \\
=\left.\frac{x^{m+\sqrt{c} l}(1-x)^{n-m}}{m+\sqrt{c} \imath}\right|_{0} ^{1} \\
\quad+\frac{n-m}{m+\sqrt{c} \imath} \int_{0}^{1} x^{m+\sqrt{c} \imath}(1-x)^{n-m-1} d x \\
=\frac{(n-m) !}{\prod_{k=m}^{n-1}(k+\sqrt{c} \imath)} \int_{0}^{1} x^{n-1+\sqrt{c} l} d x \\
=\frac{(n-m) !}{\prod_{k=m}^{n}(k+\sqrt{c} l)} .
\end{aligned}
$$

Secondly, by expanding

$$
\begin{aligned}
\int_{0}^{1} & x^{m-1+\sqrt{c} l}(1-x)^{n-m} d x \\
& =\int_{0}^{1} \sum_{k=0}^{n-m}(-1)^{k}\left(\begin{array}{c}
n-m \\
k
\end{array}\right) x^{m-1+k+\sqrt{c} l} d x \\
& =\sum_{k=0}^{n-m} \frac{(-1)^{k}\left(\begin{array}{c}
n-m \\
k
\end{array}\right)}{m+k+\sqrt{c} i} .
\end{aligned}
$$

On one hand, put such complex number in Cartesian form, and after multiplying it by $L_{m, n}^{\prime}$, we get a linear combination of $x_{k}+y_{k} \sqrt{c} \imath$ with integer coefficients, where $0 \leqslant k \leqslant$ $n-m$ and $x_{k}, y_{k} \in \mathbb{Z}$. On the other hand, it is easy to see that the number obtained by integrating by parts $(n-m)$ times is not zero. So, its modulus is not smaller than 1 , and we conclude that

$$
L_{m, n}^{\prime} \geqslant \frac{\prod_{k=m}^{n} \sqrt{k^{2}+c}}{(n-m) !} .
$$

Now, we write furthermore

$$
L_{m, n}^{\prime} \geqslant \frac{n !}{(m-1) !(n-m) !}=m\left(\begin{array}{c}
n \\
m
\end{array}\right) .
$$

Suppose now that $m \leqslant m^{\prime}:=\lceil n / 2\rceil$, then $L_{m, n}^{\prime} \geqslant L_{m^{\prime}, n}^{\prime} \geqslant$ $m^{\prime}\left(\begin{array}{c}n \\ m^{\prime}\end{array}\right)$ and $n=2 m^{\prime}$ or $2 m^{\prime}-1$.

The following Stirling estimate

$$
\left(\frac{n}{e}\right)^{n} \sqrt{2 \pi n} \leqslant n ! \leqslant\left(\frac{n}{e}\right)^{n} \sqrt{2 \pi n} e^{1 /(12 n)}
$$


allows us to obtain

$$
\left(\begin{array}{c}
2 n \\
n
\end{array}\right) \geqslant \frac{2^{2 n}}{\sqrt{n \pi}} e^{-1 /(6 n)}
$$

If $n=2 m^{\prime}$, then

$$
m^{\prime}\left(\begin{array}{c}
2 m^{\prime} \\
m^{\prime}
\end{array}\right) \geqslant 2^{2 m^{\prime}} \sqrt{\frac{m^{\prime}}{\pi}} e^{-1 /\left(6 m^{\prime}\right)} .
$$

As the function $\sqrt{(x / \pi)} e^{-1 /(6 x)}$ is increasing and its value at $x=4$ is $\geqslant 1.08$, we conclude that for any integer $m^{\prime} \geqslant 4$

$$
m^{\prime}\left(\begin{array}{c}
2 m^{\prime} \\
m^{\prime}
\end{array}\right) \geqslant 2^{2 m^{\prime}} .
$$

If $n=2 m^{\prime}-1$, then we can conclude similarly for $m^{\prime} \geqslant 5$

$$
\begin{aligned}
m^{\prime}\left(\begin{array}{c}
2 m^{\prime}-1 \\
m^{\prime}
\end{array}\right) & \geqslant\left(2 m^{\prime}-1\right)\left(\begin{array}{c}
2\left(m^{\prime}-1\right) \\
m^{\prime}-1
\end{array}\right) \\
& \geqslant 2^{2 m^{\prime}-1} \frac{m^{\prime}-1 / 2}{\sqrt{m^{\prime} \pi}} e^{-1 /\left(6 m^{\prime}\right)} \\
& \geqslant 2^{2 m^{\prime}-1} .
\end{aligned}
$$

In brief, for any integer $m, n$ with $n \geqslant 8$ and $m \leqslant\lceil n / 2\rceil$, we just establish that

$$
L_{m, n}^{\prime} \geqslant 2^{n}
$$

The case $n=7$ can be checked directly as $4\left(\begin{array}{l}7 \\ 4\end{array}\right) \geqslant 2^{7}$. For the case $n=6$, we have

$$
\begin{aligned}
{[16+c, 25+c, 36+c] } & \geqslant[25+c, 36+c] \\
& =\frac{(25+c)(36+c)}{\operatorname{gcd}(25+c, 36+c)} \\
& \geqslant \frac{25 \times 36}{11} \\
& \geqslant 64=2^{6} .
\end{aligned}
$$

For the case $n=5$, we have

$$
\begin{aligned}
{[9+c, 16+c, 25+c] } & \geqslant[16+c, 25+c] \\
& \geqslant \frac{16 \times 25}{25-16} \geqslant 2^{5} .
\end{aligned}
$$

It is easy to check the cases $n=2,3$, and 4 as it involves only two terms, and we can make our final conclusion.

\section{Acknowledgment}

The author would like to thank the referees for their helpful suggestions that improved the presentation of this paper.

\section{References}

[1] B. Green and T. Tao, "The primes contain arbitrarily long arithmetic progressions," Annals of Mathematics, vol. 167, no. 2, pp. 481-547, 2008.

[2] D. Hanson, "On the product of the primes," Canadian Mathematical Bulletin. Bulletin Canadien de Mathématiques, vol. 15, pp. 33-37, 1972.

[3] M. Nair, "On Chebyshev-type inequalities for primes," The American Mathematical Monthly, vol. 89, no. 2, pp. 126-129, 1982.

[4] H. N. Shapiro, "On the number of primes less than or equal $x$," Proceedings of the American Mathematical Society, vol. 1, pp. 346-348, 1950.

[5] B. Farhi, "Minorations non triviales du plus petit commun multiple de certaines suites finies d'entiers," Comptes Rendus Mathématique. Académie des Sciences. Paris, vol. 341, no. 8, pp. 469474, 2005.

[6] B. Farhi and D. Kane, "New results on the least common multiple of consecutive integers," Proceedings of the American Mathematical Society, vol. 137, no. 6, pp. 1933-1939, 2009.

[7] S. Hong and G. Qian, "The least common multiple of consecutive arithmetic progression terms," Proceedings of the Edinburgh Mathematical Society, vol. 54, no. 2, pp. 431-441, 2011.

[8] S. Hong and Y. Yang, "Improvements of lower bounds for the least common multiple of finite arithmetic progressions," Proceedings of the American Mathematical Society, vol. 136, no. 12, pp. 4111-4114, 2008.

[9] S. Hong and S. D. Kominers, "Further improvements of lower bounds for the least common multiples of arithmetic progressions," Proceedings of the American Mathematical Society, vol. 138, no. 3, pp. 809-813, 2010.

[10] R. Wu, Q. Tan, and S. Hong, "New lower bounds for the least common multiple of arithmetic progressions," Chinese Annals of Mathematics. In press.

[11] B. Farhi, "Nontrivial lower bounds for the least common multiple of some finite sequences of integers," Journal of Number Theory, vol. 125, no. 2, pp. 393-411, 2007.

[12] G. Qian, Q. Tan, and S. Hong, "The least common multiple of consecutive terms in a quadratic progression," Bulletin of the Australian Mathematical Society, vol. 86, pp. 389-404, 2012.

[13] S. Hong and W. Feng, "Lower bounds for the least common multiple of finite arithmetic progressions," Comptes Rendus Mathématique. Académie des Sciences. Paris, vol. 343, no. 11-12, pp. 695-698, 2006. 


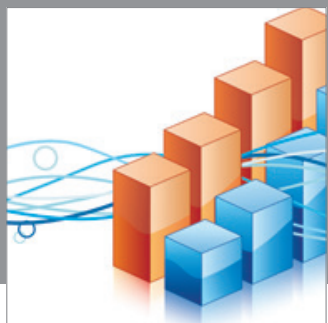

Advances in

Operations Research

mansans

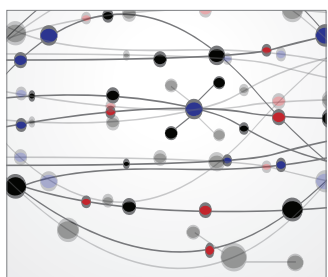

The Scientific World Journal
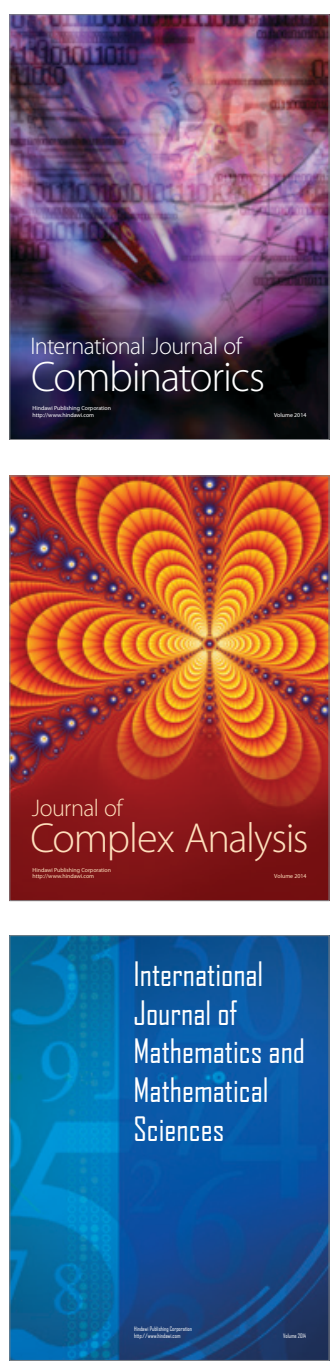
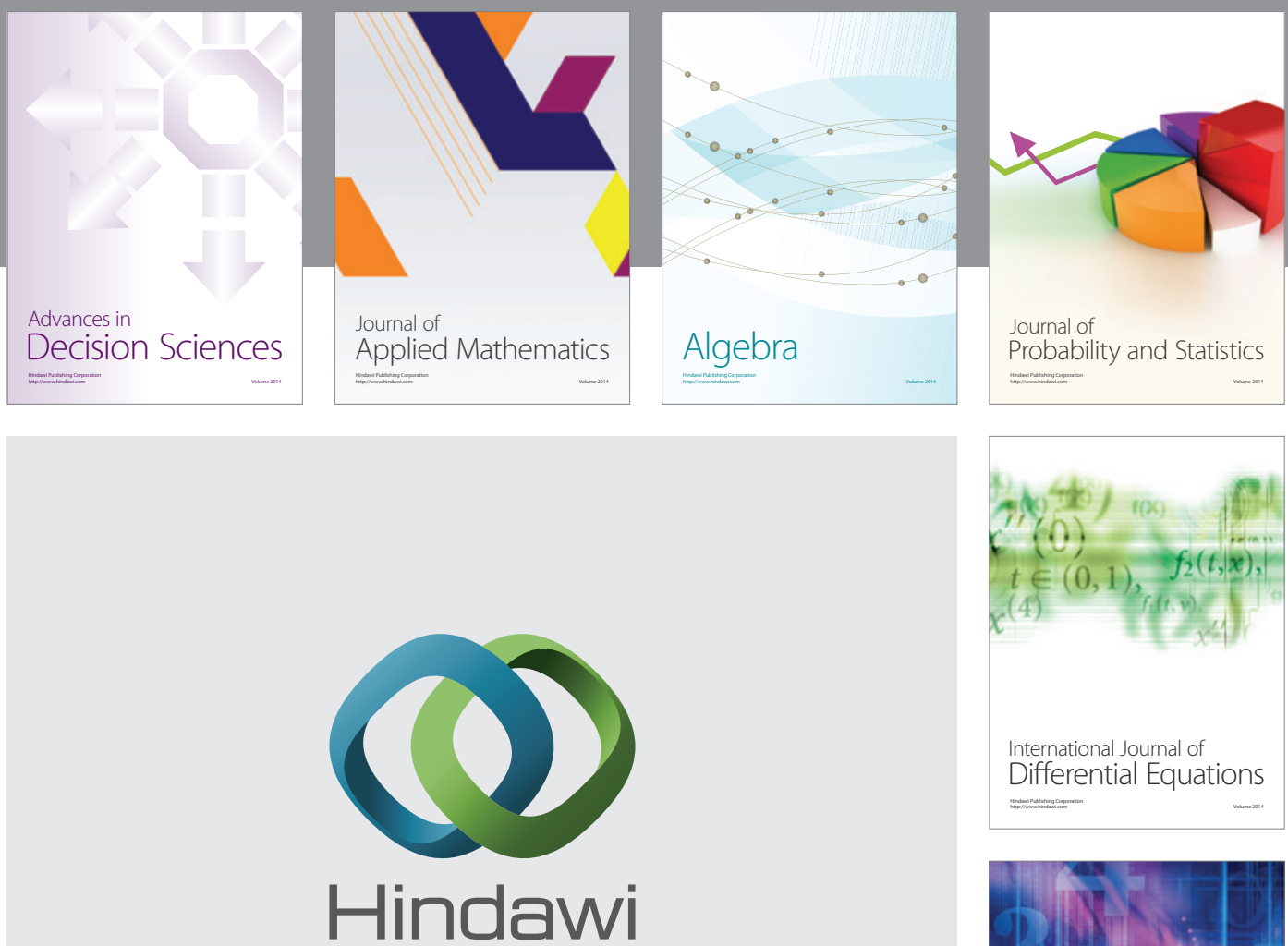

Submit your manuscripts at http://www.hindawi.com
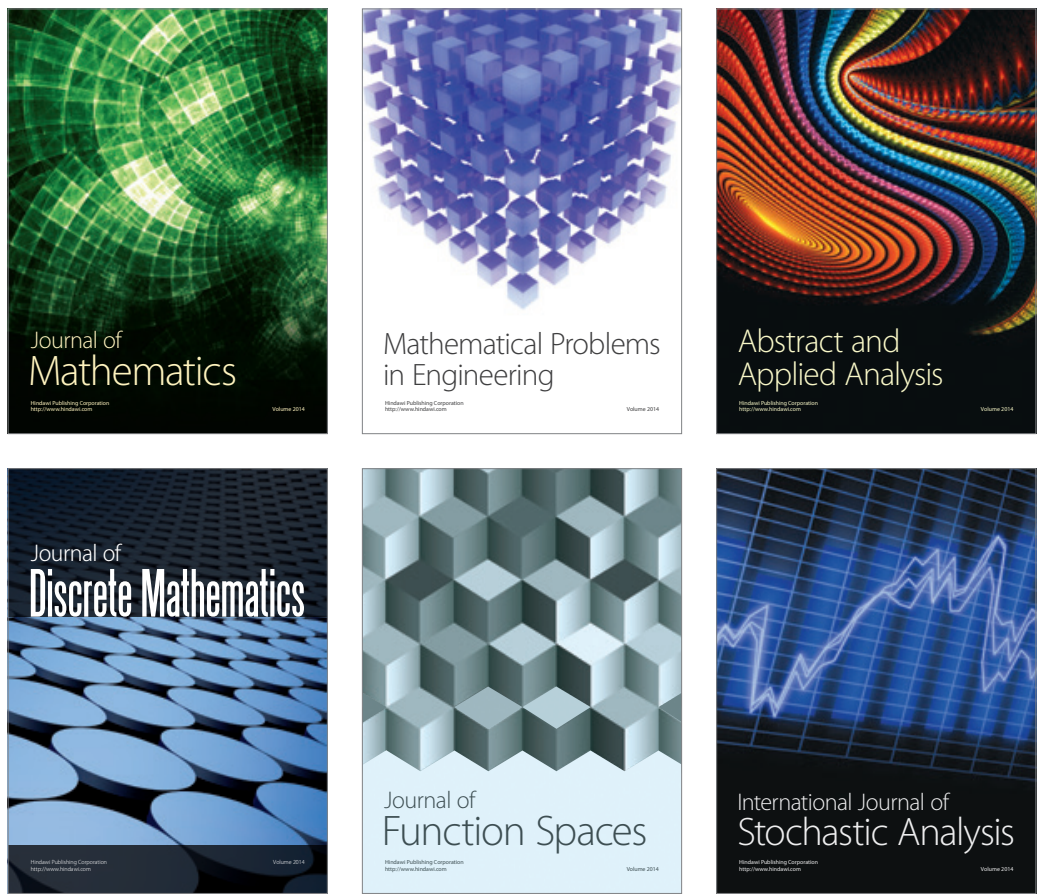

Journal of

Function Spaces

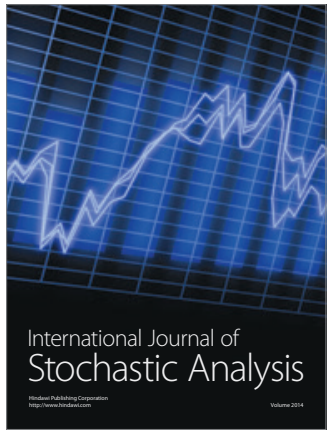

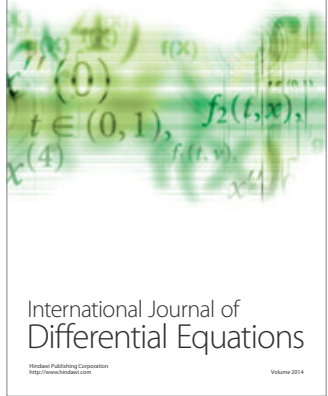
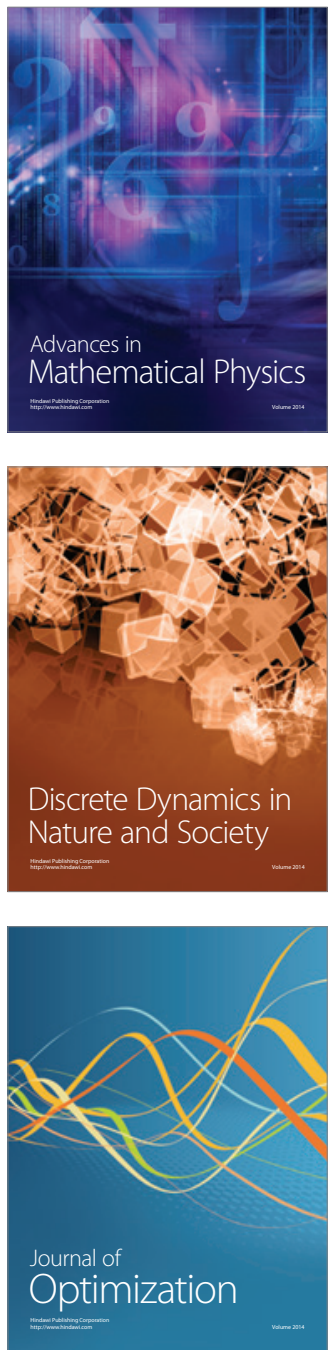